\title{
A cardinal preserving immune partition of the ordinals
}

\author{
by
}

M. C. Sta n ley (San Jose, Calif.)

\begin{abstract}
It is shown that in a cardinal and $\mathrm{GCH}$ preserving generic extension of $L$ there exists a class of ordinals that neither contains nor is disjoint from any infinite constructible set of ordinals. This answers a question of Sy Friedman's and improves a result of Shelah.
\end{abstract}

1. Introduction. An immune set (class) of ordinals is an infinite set (class) of ordinals that neither contains nor is disjoint from any infinite constructible subset of its supremum. The following result appears in Sy Friedman's paper $[\mathrm{F}]$ :

Theorem 1 (Sy Friedman). There exists an immune class of ordinals that is (class) generic over L. If $0^{\#}$ exists, then such a generic immune class of ordinals is definable over $L\left[0^{\#}\right]$.

Friedman's model is not a cardinal preserving extension of $L$, though it does satisfy the GCH. Collapsing $\aleph_{1}^{L}$ is an essential feature of his proof. The main result of the present paper settles his question whether this is an essential feature of Theorem 1 .

THEOREM 2. There exists an immune class of ordinals in a cardinal and $G C H$ preserving generic extension of $L$. If $0^{\#}$ exists, then such a generic extension of $L$ is definable over $L\left[0^{\#}\right]$.

This improves a result of Shelah. He obtained a cardinal and GCH preserving immune subset of $\aleph_{\omega}$.

Of course, it is trivial to obtain large immune sets of ordinals if the continuum hypothesis is sacrificed: The finite support product of $\kappa$ many copies of the Cohen conditions adds an unbounded immune subset of $\kappa$.

1991 Mathematics Subject Classification: 03E.

Research supported by NSF grant DMS8922393. 
Consequently, throughout we shall be interested only in models satisfying the GCH.

There are two main ideas in Sy Friedman's proof. We shall use the first and provide a cardinal preserving alternative to the second.

The first of Friedman's ideas is to consider partitions of the ordinals not just into two cells, but into larger numbers of cells. Suppose that $1<\kappa \leq \omega$. He defines a $\kappa$-partition of a set or class $X$ to be a function $f$ from $X$ into $\kappa$ and declares a $\kappa$-partition to be immune if $\left|f^{\prime \prime} z\right|=\kappa$ whenever $z$ is infinite and constructible. (So the characteristic function of an immune set is an immune 2-partition.)

Finding an immune $\omega$-partition of the ordinals suffices for Theorems 1 and 2 on account of this observation:

Lemma 1.1 (Sy Friedman). Suppose that there exists an immune $\omega$ partition of the ordinals. If $x$ is a Cohen real over $V$, then in $V[x]$ there exists an immune class of ordinals.

(If $F$ is an immune $\omega$-partition and $x: \omega \rightarrow 2$ is Cohen over $V$, then $x \circ F$ is an immune 2-partition.)

Another result appearing in $[\mathrm{F}]$ is Hugh Woodin's observation that if $0^{\#}$ exists, then, letting $\left\langle t_{i}: i<\omega\right\rangle$ enumerate all definable $L$-terms,

$$
F(\alpha)=\left\{\begin{array}{l}
\text { the least } i \text { such that } \alpha=t_{i}^{L}\left(\iota_{1}, \ldots, \iota_{k}\right) \\
\text { for some Silver indiscernibles } \iota_{1}, \ldots, \iota_{k}
\end{array}\right.
$$

is an immune $\omega$-partition of the ordinals. Unlike Sy Friedman's generic immune $\omega$-partition, $0^{\#} \in L[F]$.

A second question of Friedman's is whether it is possible to prove (in class theory) that the existence of an immune $\omega$-partition implies the existence of an immune 2-partition. With a little care, this is settled in the course of proving Theorem 2.

THEOREM 3. There exists an immune $\omega$-partition of the ordinals in a cardinal and $G C H$ preserving generic extension of $L$ that contains no immune reals. If $0^{\#}$ exists, then such a generic extension of $L$ is definable over $L\left[0^{\#}\right]$.

The utility of $\omega$-partitions is found in

Lemma 1.2 (Sy Friedman). Suppose $\lambda$ is an L-singular ordinal and that there exists an immune $\omega$-partition of each $\alpha<\lambda$. Then there exists an immune $\omega$-partition of $\lambda$.

Since we shall be using this fact, we provide a proof for the reader's convenience.

Pr o o f. Set $\mu=\operatorname{cf}^{L}(\lambda)$. Let $\left\langle\alpha_{i}: i<\mu\right\rangle$ be a constructible monotonically increasing sequence of ordinals cofinal in $\lambda$ with $\alpha_{0}=0$. Let $g$ be an immune 
$\omega$-partition of $\mu$, and let $f_{\alpha_{i}}$ be an immune $\omega$-partition of $\alpha_{i}$, for each $i<\mu$. Define $f: \lambda \rightarrow \omega$ by

$$
f(\xi)=\left\langle\left\langle f_{\alpha_{i+1}}(\xi), g(i)\right\rangle\right\rangle,
$$

where $i<\mu$ is such that $\xi \in\left[\alpha_{i}, \alpha_{i+1}\right)$ and $\langle\langle a, b\rangle\rangle=2^{a} \cdot 3^{b}$.

Suppose that $y \subseteq \lambda$ is infinite and constructible. Then $\bar{y} \subseteq \mu$ is constructible, where

$$
i \in \bar{y} \quad \text { iff } \quad y \cap\left[\alpha_{i}, \alpha_{i+1}\right) \neq \emptyset .
$$

Since $g$ is an immune $\omega$-partition of $\mu$, we may assume that $\bar{y}$ is finite. Then there exists an $i$ such that $y \cap\left[\alpha_{i}, \alpha_{i+1}\right)$ is infinite. Since $f_{\alpha_{i+1}}$ is an immune $\omega$-partition, it follows that $f$ " $y$ is infinite.

Friedman adds a generic immune $\omega$-partition of the ordinals with a backwards Easton support iteration. At each regular cardinal $\kappa$ he forces with initial segments of an immune $\omega$-partition of $\kappa$. The difficulty he must overcome is securing $<\kappa$-distributivity. Specifically, if $\left\langle q_{i}: i<\tau\right\rangle$ is a descending sequence of conditions chosen to meet $\tau$ many predense sets, measures must be taken to insure that $\bigcup_{i<\lambda} q_{i}$ is an immune $\omega$-partition when $\lambda$ is a limit ordinal of cofinality $\omega$. (If countable limits can be handled, then uncountable limits will be automatic.) Friedman's second main idea is to add $C_{\kappa}$, a generic closed unbounded subset of $\kappa$ such that every limit point of $C_{\kappa}$ has uncountable cofinality in $L$. Then, following $C_{\kappa}$, the descending sequence of conditions $\left\langle q_{i}: i<\tau\right\rangle$ can be chosen to outpace every constructible $\omega$-sequence at limits.

Of course, this strategy requires collapsing $\omega_{1}^{L}$.

In the proof of Theorems 2 and 3, we shall also use a backwards Easton support iteration, successively adding immune $\omega$-partitions of regular cardinals. When we define a descending sequence of conditions $\left\langle p_{i}: i<\tau\right\rangle$ towards constructing a condition meeting $\tau$ many predense sets, we shall not attempt to outpace every constructible $\omega$-sequence $y$ of ordinals, as did Friedman. Instead, we shall make non-trivial moves so infrequently that if the value of the generic $\omega$-partition on $y$ is not determined by some successor condition $p_{i+1}$, then $y$ must have an infinite constructible subset $y^{\prime}$ with the property that the value of the generic $\omega$-partition on $y^{\prime}$ is determined exclusively at trivial steps. The trivial steps are entirely under our control, rather than the control of the predense sets we are seeking to meet. Consequently, we can insure that the image of $y$ under the generic $\omega$-partition is infinite.

Unfortunately, I do not know how to use this idea at each step in the iteration to obtain the necessary distributivity properties for the whole iteration. Rather, it will be used on sequences of conditions in the full iteration. As usual, we shall show that a tail of the iteration is appropriately distributive in an extension by an initial segment of the iteration. The problem is that immunity is a property with respect to $L$, rather than such extensions 
of $L$. This is the main technical obstacle to the proof. It is overcome by carrying out the distributivity construction in $L$. To do this we must restrict ourselves to well-behaved conditions in the iteration and, simultaneously, prove that such conditions are dense in the full iteration.

The "infrequent moves" mentioned above are at steps indexed by elements of a sparse subset of $\tau$.

Definition. Suppose $\tau$ is a cardinal. Then $x \subseteq \tau$ is sparse iff $x$ is unbounded in $\tau$ and whenever $y \subseteq \tau$ is constructible and infinite, there exists an infinite constructible $y^{\prime} \subseteq y$ such that $y^{\prime} \cap x=\emptyset$.

For example, a Mathias real is a sparse subset of $\omega$. However, Mathias forcing adds immune reals. (If $x \subseteq \omega$ is Mathias, then $\{n:|x \cap n|$ is even $\}$ is immune.) Towards proving Theorem 3, we shall prove that certain Sacks reals are sparse and that Sacks forcing adds no immune reals.

2. Sparse and immune reals. The main tasks of this section are showing that Sacks forcing adds no immune reals over $L$ and that there exist Sacks reals that are sparse subsets of $\omega$.

To begin, let us fix our notation. The constructible Sacks conditions $\mathbb{S}$ consist of all constructible perfect subtrees $T \subseteq 2^{<\omega}$. They are ordered by set inclusion. If $G \subseteq \mathbb{S}$ is $\mathbb{S}$ generic over $L$, let $r_{G}$ denote the canonical Sacks real added, namely,

$$
r_{G}=\{n \in \omega: \exists T \in G \forall s \in T(n \in \operatorname{dom}(s) \Rightarrow s(n)=1)\} .
$$

Let us turn first to our second task. In general, Sacks reals are not sparse. Indeed, if $x \subseteq \omega$ is co-infinite and constructible and we set

$$
T_{x}=\left\{s \in 2^{<\omega}: n \in x \cap \operatorname{dom}(s) \Rightarrow s(n)=1\right\},
$$

then $T_{x}$ is a constructible Sacks condition and $T_{x} \Vdash \check{x} \subseteq \stackrel{\circ}{r}_{G}$.

LEMma 2.1. There exists a constructible Sacks condition $T_{*}$ such that

$$
T_{*} \Vdash " \stackrel{\circ}{r}_{G} \text { is sparse". }
$$

Proof. Work in $L$. Let $s \mapsto\ulcorner s\urcorner$ be a one-to-one function from $2^{<\omega}$ into $\omega$. For $s \in 2^{<\omega}$, set

$$
Z_{s}=\left\{\ulcorner t\urcorner: t \in 2^{<\omega} \text { and } s \subseteq t\right\} .
$$

The properties this secures are that $Z_{s}$ is infinite, that $Z_{\bar{s}} \supseteq Z_{s}$ when $\bar{s} \subseteq s$, and that $Z_{s \frown 0} \cap Z_{s \wedge 1}=\emptyset$.

Define a sequence $\left\langle t_{s}: s \in 2^{<\omega}\right\rangle$ of nodes in $2^{<\omega}$ by recursion on $s$ : Set $t_{\emptyset}=\emptyset$. To define $t_{s \frown i}$, choose $n$ to be least in $Z_{s^{\wedge} i} \backslash \operatorname{dom}\left(t_{s}\right)$ and set

$$
t_{s \frown i}= \begin{cases}t_{s}(k) & \text { if } k \in \operatorname{dom}\left(t_{s}\right), \\ 0 & \text { if } k \in\left[\operatorname{dom}\left(t_{s}\right), n\right), \\ 1 & \text { if } k=n .\end{cases}
$$


Set $T_{*}=\left\{t \in 2^{<\omega}: t \subseteq t_{s}\right.$ for some $\left.s \in 2^{<\omega}\right\}$. Then $T_{*}$ is a Sacks condition.

Suppose now that $G$ is $\mathbb{S}$ generic over $L$ with $T_{*} \in G$. Let $r: \omega \rightarrow 2$ be the characteristic function of the Sacks real $r_{G} \subseteq \omega$. Note first that for $s \in 2^{<\omega}$, we have

$t_{s} \subseteq r \Rightarrow r_{G}$ is eventually contained in $Z_{s}$, i.e., $\exists k\left(\left(r_{G} \backslash k\right) \subseteq Z_{s}\right)$, and

$t_{s} \not \subset r \Rightarrow r_{G}$ is eventually disjoint from $Z_{s}$, i.e., $\exists k\left(\left(r_{G} \backslash k\right) \cap Z_{s}=\emptyset\right)$.

Suppose now that $x \subseteq \omega$ is infinite and constructible and that $x \cap r_{G}$ is infinite. Then

$$
t_{s} \subseteq r \Rightarrow x \cap Z_{s} \text { is infinite. }
$$

It must be, then, that there exists a $t_{s} \not \subset$ such that $x \cap Z_{s}$ is infinite. Otherwise,

$$
t_{s} \subseteq r \Leftrightarrow x \cap Z_{s} \text { is infinite, }
$$

contradicting that $r \notin L$.

Fix a $t_{s} \nsubseteq \mathbb{r}$ such that $x \cap Z_{s}$ is infinite. Then $x \cap Z_{s}$ is an infinite constructible set such that $r_{G} \cap\left(x \cap Z_{s}\right)$ is finite. Thus there exists an infinite constructible $y \subseteq x$ such that $y \cap r_{G}=\emptyset$.

If $T \subseteq 2^{<\omega}$ is a tree, we say that $t \in T$ is a branching node when both of $t^{\frown} 0$ and $t^{\frown} 1$ lie in $T$. Define $(T)_{n}$ to be the initial segment of $T$ consisting of those nodes $t \in T$ such that $t$ has at most $n$ proper initial segments that are branching nodes. (Thus $(T)_{0}=\operatorname{stem}(T)$.) An easy induction on $n$ shows that $(T)_{n}$ is finite, for all $n$. Define $T^{\prime} \leq_{n} T$ to mean that $T^{\prime} \subseteq T$ and $\left(T^{\prime}\right)_{n}=(T)_{n}$. The following facts regarding Sacks forcing are standard.

Fusion Lemma. Suppose $\left\langle T_{n}: n \in \omega\right\rangle$ is a sequence of perfect trees such that $T_{n+1} \leq_{n} T_{n}$ for each $n$. Then $T=\bigcap_{n \in \omega} T_{n}$ is a perfect tree and $T \leq_{n} T_{n}$ for all $n$.

Lemma 2.2. Suppose $\left\langle A_{n}: n \in \omega\right\rangle$ is a sequence of antichains in the Sacks conditions $\mathbb{S}$ and that $T \in \mathbb{S}$. Then there exists a Sacks condition $T^{\prime} \leq T$ such that $\left|A_{n}\right| T^{\prime} \mid \leq 2^{n}$ for all $n$.

If $X$ is a subset of a partial ordering and $p$ is an element of that ordering, we write $X\lceil p$ to denote the collection of those elements of $X$ that are compatible with $p$.

LEMma 2.3. Work in L. Suppose that $\mu$ is an uncountable regular cardinal and that $\left\langle X_{i}: i<\omega\right\rangle$ is a tower of countable elementary substructures of $L_{\mu}$, that is,

(1) $X_{i}$ is countable;

(2) $X_{i} \prec X_{j} \prec L_{\mu}$ for $i<j<\omega$; and

(3) $X_{i} \cup\left\{X_{i}\right\} \subseteq X_{i+1}$. 
Suppose as well that the Sacks condition $T \in X_{0}$. Then there exists a condition $T^{\prime} \in \mathbb{S}$ with $T^{\prime} \leq T$ such that if $G \subseteq \mathbb{S}$ is generic with $T^{\prime} \in G$, then

$$
X_{i}[G] \prec X_{j}[G] \prec L_{\mu}[G]
$$

for $i<j<\omega$.

This section's first task remains:

LEMMA 2.4. Sacks forcing adds no immune reals over $L$.

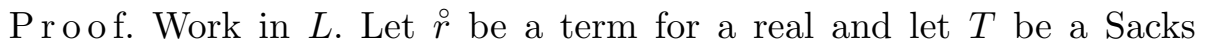
condition. We may assume that

$$
\left\{n \in \omega: T^{\prime} \Vdash \check{n} \in \stackrel{\circ}{r}\right\} \text { is finite for all } T^{\prime} \leq T .
$$

Define an increasing sequence of natural numbers $\left\langle n_{i}: i<\omega\right\rangle$ and a fusion sequence $\left\langle T_{i}: i<\omega\right\rangle$ such that $T_{i} \Vdash \check{n}_{i} \notin \stackrel{\circ}{r}$ by recursion on $i$ : Set $T_{0}=T$ and

$$
n_{i}=\left\{\begin{array}{c}
\text { the least } n>n_{j}, \text { for all } j<i, \text { such that } \\
T_{i} \uparrow t \forall \check{n} \in \dot{r} \text { for all } t \in\left(T_{i}\right)_{i} .
\end{array}\right.
$$

(Here $T_{i} \uparrow t=\left\{s \in T_{i}: t \subseteq s\right.$ or $\left.s \subseteq t\right\}$.) Using (2.1) and that $\left(T_{i}\right)_{i}$ is finite, there exists such an $n$. To define $T_{i+1}$, let $X \subseteq\left(T_{i}\right)_{i}$ comprise all $\subseteq$-maximal nodes and choose $T_{t} \leq T_{i} \backslash t$ such that $T_{t} \Vdash \check{n}_{i} \notin \stackrel{r}{r}$ for each $t \in X$. Then set $T_{i+1}=\bigcup_{t \in X} T_{t}$.

Note that $T_{i+1} \leq_{i} T_{i}$ and that $T_{i+1} \Vdash \check{n}_{i} \notin \stackrel{\circ}{ }$. Set $T^{\prime}=\bigcap_{i \in \omega} T_{i}$. Then $T^{\prime} \Vdash \check{n}_{i} \notin \stackrel{\circ}{r}$ for all $i$.

Re mark. An alternative to Sacks forcing is sparse tree forcing. We say that a tree $T \subseteq 2^{<\omega}$ is sparse if $t^{\frown} 0 \in T$ whenever $t^{\frown} 1 \in T \backslash \operatorname{stem}(T)$. The sparse tree conditions consist of all sparse perfect trees $T \subseteq 2^{<\omega}$, ordered by set inclusion.

With sparse tree forcing it is not necessary to work below a special condition to obtain a sparse real. In fact, if $r_{G} \subseteq \omega$ is sparse-tree-generic over $L$, then $r_{G}$ has the following property which is strictly stronger than sparseness:

Suppose $F: \omega \rightarrow \omega$ is constructible and non-decreasing. Then there exists an infinite constructible $y \subseteq \omega$ such that $y \cap F^{\prime \prime} r_{G}=\emptyset$.

(No real in a Sacks extension of $L$ enjoys this property because in such a model every $f: \omega \rightarrow \omega$ is dominated by a constructible function $g: \omega \rightarrow \omega$.) The Fusion Lemma and Lemmas 2.2-2.4 hold for sparse tree forcing by essentially the same proofs.

3. Sparse sets of ordinals. Suppose that $\kappa>\omega$ is regular. Let $\mathbb{Q}_{\kappa}$ consist of all functions $q: \alpha \rightarrow 2$, for some $\alpha<\kappa$, such that if $x$ is an infinite 
constructible set of ordinals, then there exists an infinite constructible $y \subseteq x$ such that $y \cap q^{-1}(1)=\emptyset$. Let $\mathbb{Q}_{\kappa}$ be ordered by reverse inclusion, that is, by reverse functional extension.

Lemma 3.1. Suppose that $\bar{q} \in \mathbb{Q}_{\kappa}$ and that $\operatorname{dom}(\bar{q})<\delta<\kappa$. Then there exists a condition $q$ extending $\bar{q}$ in $\mathbb{Q}_{\kappa}$ such that $q(\delta)=1$.

Proof. Define $q$ with $\operatorname{dom}(q)=\delta+1$ by setting $q(\xi)=0$ for $\xi \epsilon$ $[\operatorname{dom}(\bar{q}), \delta)$, and setting $q(\delta)=1$.

If $G \subseteq \mathbb{Q}_{\kappa}$ is generic, set

$$
Q_{\kappa}=\{\delta<\kappa: q(\delta)=1 \text { for some } q \in G\} .
$$

Then $Q_{\kappa} \subseteq \kappa$ is sparse, since $\kappa>\omega$ is regular in $L$. What is not evident is that forcing with $\mathbb{Q}_{\kappa}$ preserves cardinals. In fact, if there exists a sparse $Q_{\tau} \subseteq \tau$ for each regular $\tau<\kappa$, then $\mathbb{Q}_{\kappa}$ is $<\kappa$-distributive. A proof of this is implicit in the proof of the analogous fact for the iteration $\widehat{\mathbb{P}}$ in $\S 5$. Since it is unnecessary, we shall not give an explicit proof.

4. Immune $\omega$-partitions of sets. Suppose that $\kappa>\omega$ is regular. Let $\mathbb{R}_{\kappa}$ consist of all functions $r: \alpha \rightarrow \omega$, for some $\alpha<\kappa$, such that if $x \subseteq \alpha$ is infinite and constructible, then $r$ " $x$ is infinite. Let $\mathbb{R}_{\kappa}$ be ordered by reverse set inclusion, that is, by reverse functional extension.

LEMMA 4.1. Assume that there exists an immune $\omega$-partition of $\tau$ for each infinite $L$-cardinal $\tau<\kappa$. Suppose that $\bar{r} \in \mathbb{R}_{\kappa}$ and that $\delta<\kappa$. Then there exists a condition $r$ extending $\bar{r}$ in $\mathbb{R}_{\kappa}$ such that $\delta \subseteq \operatorname{dom}(r)$.

Proof. Begin by noting that there exists an immune $\omega$-partition of $\delta$. Indeed, if $g:|\delta|^{L} \rightarrow \omega$ is an immune partition of $|\delta|^{L}$ and $h: \delta \leftrightarrow|\delta|^{L}$ is a constructible bijection, then $g \circ h$ is an immune $\omega$-partition of $\delta$.

Let $f: \delta \rightarrow \omega$ be an immune $\omega$-partition and set $r=\bar{r} \cup(f \uparrow[\operatorname{dom}(\bar{r}), \delta))$. Then $r$ is as required.

It follows that if there exists an immune $\omega$-partition of each infinite $L$-cardinal $\tau<\kappa$, and if $G \subseteq \mathbb{R}_{\kappa}$ is generic, then $R_{\kappa}=\bigcup G$ is an immune $\omega$-partition of $\kappa$. Again, it is not evident that forcing with $\mathbb{R}_{\kappa}$ preserves cardinals. Though, again, we shall not need this fact explicitly, if also there exists a sparse subset of $\tau$, for each regular $\tau<\kappa$, then $\mathbb{R}_{\kappa}$ is $<\kappa$-distributive.

5. The iteration. Work in $L$. Let CARD denote the class of infinite cardinals. If $A, B \subseteq 2^{<\alpha}$, it will be convenient to let $A \times B$ comprise only pairs $(a, b)$ with $a \in A$ and $b \in B$ such that $\operatorname{dom}(a)=\operatorname{dom}(b)$. Let ht $((a, b))$ denote $\delta$, where $\delta$ is the common domain of $a$ and $b$. 
If $\kappa$ is an infinite cardinal, let $\widehat{\mathbb{P}}^{\kappa}$ denote the backwards Easton support iteration of Sacks forcing below a fixed condition forcing the generic real to be sparse, followed by $\mathbb{Q}_{\alpha} \times \mathbb{R}_{\alpha}$ for uncountable regular $\alpha<\kappa$. We may identify conditions in $\widehat{\mathbb{P}}^{\kappa}$ with functions $p$ satisfying the following requirements:

(1) $\operatorname{sp}(p) \subseteq \mathrm{CARD} \cap \kappa$ and $|\operatorname{sp}(p) \cap \tau|<\tau$ for all regular $\tau$, where $\operatorname{sp}(p)$ denotes the domain of the function $p$.

(2) Suppose that $\alpha \in \operatorname{sp}(p)$.

(a) If $\alpha=\omega$, then $p(\alpha) \in \mathbb{S}$ and $p(\alpha) \leq T_{*}$, where $T_{*}$ is a fixed condition forcing the Sacks real to be sparse;

(b) $p(\alpha)=\emptyset$ if $\alpha$ is singular; and

(c) $p(\alpha)$ is a $\widehat{\mathbb{P}}^{\alpha}$-term such that $\widehat{\mathbb{P}}^{\alpha} \Vdash p(\alpha) \in \mathbb{Q}_{\alpha} \times \mathbb{R}_{\alpha}$ if $\alpha$ is uncountable and regular.

Set $\widehat{\mathbb{P}}=\bigcup_{\kappa \in \mathrm{CARD}} \widehat{\mathbb{P}}^{\kappa}$.

In clause $(2 \mathrm{a})$ we can let $T_{*}$ be the Sacks condition constructed in $\S 2$. Then $\widehat{\mathbb{P}}$ is definable without parameters in $L$.

As usual, $\widehat{\mathbb{P}}^{\kappa}$ is ordered by

$$
\begin{array}{ll}
\bar{p} \geq p \quad \text { iff } \quad & \operatorname{sp}(\bar{p}) \subseteq \operatorname{sp}(p) \text { and } \\
& p\lceil\alpha \Vdash \bar{p}(\alpha) \geq p(\alpha) \text { for all regular } \alpha \in \operatorname{sp}(\bar{p}) .
\end{array}
$$

The main fact to be proved about $\widehat{\mathbb{P}}^{\kappa}$ is that if $\tau<\kappa$ is regular, then $\widehat{\mathbb{P}}^{\tau^{+}}$forces that $\widehat{\mathbb{P}}_{\tau^{+}}^{\kappa}$ is $\leq \tau$-distributive, where $\widehat{\mathbb{P}}_{\tau^{+}}^{\kappa}$ is the tail of the iteration beginning at $\tau^{+}$. To do this, we shall define a descending $\tau$-sequence of conditions in $\widehat{\mathbb{P}}_{\tau^{+}}^{\kappa}$ below a given condition, gradually meeting $\tau$ many predense sets in $\widehat{\mathbb{P}}_{\tau^{+}}^{\kappa}$. Of course, if $p$ is a limit condition in this process and $\alpha$ is an uncountable regular cardinal in the support of $p$, then $p(\alpha)$ must be a term for a condition in $\mathbb{Q}_{\alpha} \times \mathbb{R}_{\alpha}$. Conditions in $\mathbb{Q}_{\alpha}$ are approximations to a sparse subset of $\alpha$. The twist is that sparseness is a requirement with respect to $L$ rather than $L[G]$, where $G$ is $\widehat{\mathbb{P}}^{\tau^{+}}$generic. And it is in $L[G]$ that this distributivity construction is naturally described. A similar observation applies to $\mathbb{R}_{\alpha}$.

One way out of this is to carry out the distributivity construction following elementary towers that are definable in $L$. Most of our work will be with a sub-ordering $\mathbb{P}^{\kappa}$ of $\widehat{\mathbb{P}}^{\kappa}$ that comprises the conditions in $\widehat{\mathbb{P}}^{\kappa}$ that are pointwise of low rank. We shall argue simultaneously by induction on $\kappa$ that $\mathbb{P}^{\kappa}$ has the distributivity property mentioned above and that $\mathbb{P}^{\kappa}$ is dense in $\widehat{\mathbb{P}}^{\kappa}$.

Define $\mathbb{P}^{\kappa}$ to consist of those conditions $p \in \widehat{\mathbb{P}}^{\kappa}$ satisfying the following two additional requirements: 
$\left(2 \mathrm{c}^{\prime}\right) p(\alpha)$ is a $\mathbb{P}^{\alpha}$-term (rather than merely a $\widehat{\mathbb{P}}^{\alpha}$-term) and $\mathbb{P}^{\alpha} \Vdash p(\alpha) \in$ $\mathbb{Q}_{\alpha} \times \mathbb{R}_{\alpha}$ if $\alpha$ is uncountable and regular.

(3) If $\alpha \in \operatorname{sp}(p)$ and $\alpha>\omega$, then $p(\alpha) \in L_{\alpha}$.

Then $\mathbb{P}$ is definable without parameters in $L$.

The reason that it is not obvious that $\mathbb{P}^{\kappa}$ is dense in $\widehat{\mathbb{P}}^{\kappa}$ is that $\mathbb{P}^{\lambda}$ has antichains of size $\lambda^{+}$when $\lambda$ is singular.

Note that

(5.1) $\quad \mathbb{P}^{\kappa} \subseteq L_{\kappa^{+}}$for all infinite cardinals $\kappa$;

(5.2) if $\kappa>\omega$ is regular, then $\mathbb{P}^{\kappa^{+}} \subseteq L_{\kappa}$ and $\mathbb{P}^{\kappa^{+}} \in L_{\beta}$ if $\beta>\kappa$ is a $\mathrm{ZF}^{-}$-ordinal; and if $\kappa>\omega$ is singular, then $\mathbb{P}^{\kappa}$ is isomorphic to a dense open subset of $\mathbb{P}^{\kappa^{+}}$;

(5.3) if $\kappa$ is inaccessible, then $\mathbb{P}^{\kappa} \subseteq L_{\kappa}$; and

(5.4) $\quad \mathbb{P}^{\omega_{1}} \subseteq L_{\omega_{1}}$ and $\mathbb{P}^{\omega_{1}} \in L_{\beta}$ if $\beta>\omega_{1}$ is a $\mathrm{ZF}^{-}$-ordinal.

Let us summarize the cardinality analogs of these observations, towards verifying that $\widehat{\mathbb{P}}$ is cardinal and GCH preserving.

Lemma 5.1. Suppose that $\kappa$ is an infinite cardinal. Then

(a) $\left|\mathbb{P}^{\kappa^{+}}\right|=\kappa$ if $\kappa>\omega$ is regular.

(b) $\left|\mathbb{P}^{\kappa}\right|=\kappa$ if $\kappa=\omega_{1}$, or if $\kappa$ is inaccessible, or if $\kappa$ is the successor of a singular cardinal.

(c) $\left|\mathbb{P}^{\kappa}\right|=\kappa^{+}$if $\kappa$ is singular.

Suppose that $\tau$ is regular and that $\kappa \geq \tau^{+}$is a cardinal. Set

$$
\widehat{\mathbb{P}}_{\tau^{+}}^{\kappa}=\left\{p\left\lceil\left[\tau^{+}, \kappa\right): p \in \widehat{\mathbb{P}}^{\kappa}\right\} \quad \text { and } \quad \mathbb{P}_{\tau^{+}}^{\kappa}=\widehat{\mathbb{P}}_{\tau^{+}}^{\kappa} \cap \mathbb{P}^{\kappa} .\right.
$$

Order $\widehat{\mathbb{P}}_{\tau^{+}}^{\kappa}$ (respectively, $\mathbb{P}_{\tau^{+}}^{\kappa}$ ) in $L[G]$, where $G$ is $\widehat{\mathbb{P}}^{\tau^{+}}$(respectively, $\mathbb{P}^{\tau^{+}}$) generic, by

$$
\bar{p} \geq p \quad \text { iff } \quad p^{\prime} \cup \bar{p} \geq p^{\prime} \cup p, \text { for some } p^{\prime} \in G .
$$

As mentioned above, the main fact to be proved is the

Distributivity Lemma. Suppose that $\kappa$ is an uncountable cardinal and that $\tau<\kappa$ is infinite and regular. Then

$$
\mathbb{P}^{\tau^{+}} \Vdash \text { "P } \tau_{\tau^{+}}^{\kappa} \text { is } \leq \tau \text {-distributive". }
$$

Three auxiliary facts will be needed.

Antichain Lemma. Suppose that $\kappa$ is an uncountable cardinal, that $\left\langle A_{\gamma}: \gamma\langle\alpha\rangle\right.$ is a sequence of antichains in $\widehat{\mathbb{P}}^{\kappa}$, where $\alpha<\kappa$, and that $\bar{p} \in \widehat{\mathbb{P}}^{\kappa}$. Then there exists a condition $p$ extending $\bar{p}$ such that $\left|A_{\gamma}\right| p|\leq| \alpha \mid$ for all $\gamma<\alpha$.

Recall that $A_{\gamma}\left\lceil p=\left\{p^{\prime} \in A_{\gamma}: p^{\prime}\right.\right.$ is compatible with $\left.p\right\}$.

Density Lemma. Suppose that $\kappa$ is an uncountable cardinal. 
(a) $\mathbb{P}^{\kappa}$ is dense in $\widehat{\mathbb{P}}^{\kappa}$.

(b) If $\tau<\kappa$ is uncountable and regular, and if $\bar{p} \in \widehat{\mathbb{P}}_{\tau^{+}}^{\kappa}$, then there exists a condition $p \in \mathbb{P}_{\tau^{+}}^{\kappa}$ such that $\widehat{\mathbb{P}}^{\tau^{+}} \Vdash p \leq \bar{p}$.

Note that it follows from (a) that if $\tau<\kappa$ is an infinite cardinal, then

$$
\widehat{\mathbb{P}}^{\tau^{+}} \Vdash \text { " } \mathbb{P}_{\tau^{+}}^{\kappa} \text { is dense in } \widehat{\mathbb{P}}_{\tau^{+}}^{\kappa} " .
$$

Part (b) is a stronger form of (5.5) that is useful in maintaining the inductive proof of the Density Lemma.

Extension Lemma. Suppose that $\kappa$ is an uncountable cardinal, that $\tau<\kappa$ is an infinite cardinal, that $\bar{p} \in \mathbb{P}_{\tau^{+}}^{\kappa}$, and that $f: \operatorname{CARD} \cap\left[\tau^{+}, \kappa\right) \rightarrow \kappa$ is such that $f(\alpha)<\alpha$ for all $\alpha$. If $\bar{p}\lceil\alpha \Vdash \operatorname{ht}(\bar{p}(\alpha))<f(\alpha)$ for all uncountable regular $\alpha \in \operatorname{sp}(\bar{p})$, then there exists a condition $p$ extending $\bar{p}$ in $\mathbb{P}^{\kappa}$ such that $\operatorname{sp}(p)=\operatorname{sp}(\bar{p})$ and $p\lceil\alpha \Vdash \operatorname{ht}(p(\alpha))=f(\alpha)$ for all uncountable regular $\alpha \in \operatorname{sp}(p)$.

Of course, using these lemmas, we have what we want:

COROLlary. Forcing with $\widehat{\mathbb{P}}$ preserves all L-cardinals and the GCH. In any $\widehat{\mathbb{P}}$ generic extension of $L$ there exists a sparse subset of and an immune $\omega$-partition of each infinite cardinal.

To be precise, these four lemmas are proved simultaneously by induction on $\kappa$. The following table indicates how they depend on each other.

\begin{tabular}{|l|l|}
\hline The proof at $\kappa$ of the & depends on the \\
\hline Antichain Lemma & Distributivity Lemma at $\kappa$ and Density Lemma at $\kappa$ \\
Distributivity Lemma & Extension Lemma at $\kappa$ and Density Lemma below $\kappa$ \\
Density Lemma & Extension and Antichain Lemmas below $\kappa$ \\
Extension Lemma & Density Lemma below $\kappa$ \\
\hline
\end{tabular}

Proof of the Antichain Lemma. Set $\tau=|\alpha|$. We may assume that $\tau$ is infinite.

First suppose that $\tau$ is a regular cardinal. Note that $\widehat{\mathbb{P}}^{\tau^{+}} \Vdash{ }^{\top} \widehat{\mathbb{P}}_{\tau^{+}}$is $\leq \tau$ distributive". Indeed, $\widehat{\mathbb{P}}^{\tau^{+}} \Vdash$ "PP्P $\tau^{+}$is dense in $\widehat{\mathbb{P}}_{\tau^{+}}^{\kappa}$ ", the sub-ordering $\mathbb{P}^{\tau^{+}}$is equivalent to $\widehat{\mathbb{P}}^{\tau^{+}}$, and $\mathbb{P}^{\tau^{+}} \Vdash$ " $\mathbb{P}_{\tau^{+}}^{\kappa}$ is $\leq \tau$-distributive". Also, $\widehat{\mathbb{P}} \kappa \cong \widehat{\mathbb{P}}^{\tau^{+}} * \widehat{\mathbb{P}}_{\tau^{+}}^{\kappa}$. The lemma then follows from $\left|\mathbb{P}^{\tau^{+}}\right|=\tau$ if $\tau>\omega$, and from Lemma 2.2 (countable antichain reduction in $\mathbb{S}$ ) if $\tau=\omega$.

If $\tau$ is singular, say $\operatorname{cf}(\tau)=\mu$, then let $\left\langle\beta_{i}: i<\mu\right\rangle$ be an increasing sequence of infinite regular cardinals that is cofinal in $\tau$. For each $i<\mu$, let $D_{i} \subseteq \widehat{\mathbb{P}}^{\kappa}$ be a maximal antichain of conditions $p^{\prime}$ such that $\left|A_{j}\right| p^{\prime} \mid \leq \beta_{i}$ for all $j<\beta_{i}$. Choose $p \leq \bar{p}$ such that $\mid D_{i}\lceil p \mid \leq \mu$ for all $i<\mu$. 
Proof of the Extension Lemma. For each uncountable regular $\alpha \in \operatorname{sp}(\bar{p})$, choose $\stackrel{\circ}{q}_{\alpha}$ and $\stackrel{\circ}{r}_{\alpha}$ such that

$$
\begin{gathered}
\mathbb{P}^{\alpha} \Vdash \stackrel{\circ}{q}_{\alpha} \in \mathbb{Q}_{\alpha}, \\
\bar{p}\left\lceil\alpha \Vdash \operatorname{dom}\left(\stackrel{\circ}{q}_{\alpha}\right)=f(\alpha)^{\vee},\right. \\
\mathbb{P}^{\alpha} \Vdash \stackrel{\circ}{r}_{\alpha} \in \mathbb{R}_{\alpha}, \\
\bar{p}\left\lceil\alpha \Vdash \operatorname{dom}\left(\stackrel{\circ}{\alpha}_{\alpha}\right)=f(\alpha)^{\vee},\right. \\
\mathbb{P}^{\alpha} \Vdash\left(\stackrel{\circ}{q}_{\alpha}, \stackrel{\circ}{r}_{\alpha}\right) \leq \bar{p}(\alpha) .
\end{gathered}
$$

Note first that we may assume that $\stackrel{\circ}{q}_{\alpha}, \stackrel{\circ}{r}_{\alpha} \in L_{\alpha}$. Indeed, using $\bar{p}(\alpha) \in L_{\alpha}$, it is trivial to choose $\stackrel{\circ}{q}_{\alpha} \in L_{\alpha}$ (cf. Lemma 3.1). By the proof of Lemma 4.1, an adequate $\dot{r}_{\alpha}$ can be defined in $L_{\alpha}$ from

(1) $\bar{p}(\alpha)$;

(2) an $h: f(\alpha) \leftrightarrow|f(\alpha)|^{L}$; and

(3) a term $\stackrel{\circ}{g}$ such that $\mathbb{P}^{\alpha} \Vdash " \stackrel{g}{g}$ is an immune $\omega$-partition of $|f(\alpha)|^{L}$ ",

provided that $\stackrel{\circ}{g} \in L_{\alpha}$. If $\alpha=\omega_{1}$, then (3) is trivial. If $\alpha>\omega_{1}$ is not the successor of a singular cardinal, then we have $\mathbb{P}^{|f(\alpha)|^{+}} \in L_{\alpha}$, by lines (5.1) and (5.2). Using the Density Lemma, it follows that there exists a term $\stackrel{\circ}{g} \in L_{\alpha}$ as in (3). On the other hand, if $\alpha=\lambda^{+}$, where $\lambda$ is a singular cardinal, then a term $\stackrel{\circ}{g}$ for an immune $\omega$-partition of $\lambda$ is definable from terms for immune $\omega$-partitions of $L$-cardinals less than $\lambda$ (cf. Lemma 1.2). It follows that there exists a term $\stackrel{\circ}{g} \in L_{\alpha}$ as in (3).

Let $\stackrel{\circ}{\alpha}_{\alpha} \in L_{\alpha}$ be such that $\mathbb{P}^{\alpha} \Vdash \stackrel{\circ}{z}_{\alpha}=\left(\stackrel{\circ}{q}_{\alpha}, \stackrel{\circ}{r}_{\alpha}\right)$ and set

$$
p(\alpha)= \begin{cases}\stackrel{\circ}{z}_{\alpha} & \text { if } \alpha \in \operatorname{sp}(\bar{p}) \text { is uncountable and regular } \\ \bar{p}(\alpha) & \text { if } \alpha \in \operatorname{sp}(\bar{p}) \text { is singular or } \alpha=\omega .\end{cases}
$$

Proof of the Distributivity Lemma. Suppose $\bar{p} \in \mathbb{P}^{\kappa}$ and that $\vec{D}=\left\langle\stackrel{\circ}{D}_{i}: i<\tau\right\rangle$ is a sequence of $\mathbb{P}^{\tau^{+}}$-terms for predense subsets of $\mathbb{P}_{\tau^{+}}^{\kappa}$. We may assume that $\vec{D} \in L_{\kappa^{++}}$. We may also assume that $\tau^{+}<\kappa$. Define $\left\langle X_{i}: i<\tau\right\rangle$ by setting

$$
X_{i}=\text { Skolem Hull }_{L_{\kappa++}}\left(\kappa \cup\{\kappa, \bar{p}, \vec{D}\} \cup\left\{X_{j}: j<i\right\}\right) .
$$

Next, for $\alpha^{+} \in \mathrm{CARD} \cap\left[\tau^{+}, \kappa\right)$, define

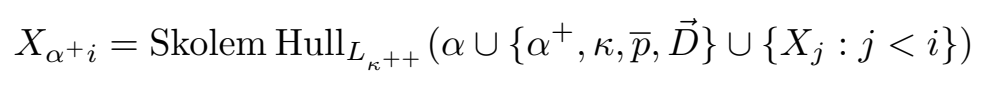

and, if $\alpha \in \operatorname{CARD} \cap\left[\tau^{+}, \kappa\right)$ is inaccessible, define

$$
X_{\alpha i}=\operatorname{Skolem~Hull}_{L_{\kappa}++}\left(\omega_{1} \cup \tau \cup\left\{\tau^{+}, \alpha, \kappa, \bar{p}, \vec{D}\right\} \cup\left\{X_{j}: j<i\right\}\right) .
$$


Note that if $i<\tau$ is a limit ordinal, then

$$
\begin{aligned}
X_{i} & =\bigcup_{j<i} X_{j}, \quad \text { and } \\
X_{\alpha i} & =\bigcup_{j<i} X_{\alpha j} \quad \text { for all regular } \alpha \in \operatorname{CARD} \cap\left[\tau^{+}, \kappa\right) .
\end{aligned}
$$

Also note that

$$
\left\langle X_{\beta j}: \beta \in \mathrm{CARD} \cap\left[\tau^{+}, \kappa\right) \text { is regular and } j \leq i\right\rangle \in X_{\alpha(i+1)}
$$

for all $\alpha$ and all $i$. This is because these Skolem hulls obey

$$
X_{\beta j} \prec X_{j} \prec X_{i} \prec L_{\kappa^{++}}
$$

and $X_{i} \in X_{\alpha(i+1)}$. Set

$$
f_{i}(\alpha)=\sup \left(X_{\alpha i} \cap \alpha\right)
$$

for $i<\tau$ and regular $\alpha \in \mathrm{CARD} \cap\left[\tau^{+}, \kappa\right)$. Then

(1) $\left\langle f_{i}(\alpha): i<\tau\right\rangle$ is a continuous, strictly increasing sequence of ordinals less than $\alpha$;

(2) $f_{i}\left(\alpha^{+}\right)=X_{\alpha^{+} i} \cap \alpha^{+}$;

(3) if $\alpha$ is inaccessible, then $f_{i}(\alpha)$ is a (singular) cardinal; and

(4) $\left\langle f_{j}: j \leq i\right\rangle \in X_{\tau^{+}(i+1)}$.

Set $f_{\tau}(\alpha)=\sup _{i<\tau} f_{i}(\alpha)$.

Note that if $p \in \mathbb{P}_{\tau^{+}}^{\kappa} \cap X_{\tau^{+} i}$, then $p\left\lceil\alpha \Vdash \operatorname{ht}(p(\alpha))<f_{i}(\alpha)\right.$ for all regular $\alpha \in \operatorname{sp}(p)$. Let us make this observation explicit, since we employ the subordering $\mathbb{P}^{\kappa}$ mainly for its sake. Fix a regular $\alpha \in \operatorname{sp}(p)$. Then $p \in X_{\alpha i}$, because the parameters from which $p$ is definable in $X_{\tau^{+i}}$ are included in $X_{\alpha i}$. Also, $\alpha \in X_{\alpha i}$, so $p(\alpha) \in X_{\alpha i}$. The term rank of $p(\alpha)$ can be calculated in $X_{\alpha i}$, and this rank is less than $f_{i}(\alpha)$, since $p(\alpha) \in L_{\alpha}$. It follows that $p\left\lceil\alpha \Vdash \operatorname{ht}(p(\alpha))<f_{i}(\alpha)\right.$.

Our next task is to define functions $g_{i}^{\alpha^{+}}$canonically projecting $f_{i}\left(\alpha^{+}\right)$ into $\alpha$ satisfying the following three requirements:

(1) $g_{i}^{\alpha^{+}}: f_{i}\left(\alpha^{+}\right) \stackrel{1: 1}{\longrightarrow} \alpha$;

(2) $g_{i}^{\alpha^{+}} \subseteq g_{j}^{\alpha^{+}}$for $i<j<\tau$; and

(3) $\left\langle g_{j}^{\alpha^{+}}: \alpha^{+} \in \mathrm{CARD} \cap\left[\tau^{+}, \kappa\right)\right.$ and $\left.j \leq i\right\rangle \in X_{\tau^{+}(i+1)}$.

Towards this, we shall need two auxiliary objects, namely an ordering $\triangleleft^{\alpha}$ and a function $h^{\alpha}$.

Suppose that $\alpha \in \operatorname{CARD} \cap[\tau, \kappa)$. Let $\alpha^{<\omega}$ denote the set of all finite increasing sequences of elements of $\alpha$. Let $\triangleleft^{\alpha}$ be the $L$-least well ordering of $\tau^{<\omega} \times \alpha^{<\omega} \times \omega$ such that

(5.6) if $\vec{\imath}, \vec{\jmath} \in \tau^{<\omega}$ and $\max (\vec{\imath})<\max (\vec{\jmath})$, then $(\vec{\imath}, \vec{\xi}, n) \triangleleft^{\alpha}(\vec{\jmath}, \vec{\zeta}, m)$ 
for all $\vec{\xi}, \vec{\zeta} \in \alpha^{<\omega}$ and all $n, m \in \omega$. Then $\left\langle\triangleleft^{\beta}: \beta \in \operatorname{CARD} \cap[\tau, \kappa)\right\rangle \in X_{\alpha i}$ for all $\alpha$ and $i$. Let $h^{\alpha}$ be the $L$-least bijection

$$
h^{\alpha}: \tau^{<\omega} \times \alpha^{<\omega} \times \omega \leftrightarrow \alpha .
$$

We use $\triangleleft^{\alpha}$ and $h^{\alpha}$ to define $g^{\alpha^{+}}: f_{\tau}\left(\alpha^{+}\right) \stackrel{1: 1}{\longrightarrow} \alpha$ as follows:

$$
g^{\alpha^{+}}(\delta)=\left\{\begin{array}{l}
h^{\alpha}\left(\left\langle i_{0}, \ldots, i_{k}\right\rangle, \vec{\xi},\ulcorner\varphi\urcorner\right), \text { where }(\vec{\imath}, \vec{\xi},\ulcorner\varphi\urcorner) \\
\text { is the } \triangleleft^{\alpha} \text {-least triple with the property that } \\
\delta \text { is the least ordinal such that } \\
\quad \varphi^{L_{\kappa}++}\left[\delta, X_{i_{0}}, \ldots, X_{i_{k}}, \vec{\xi}, \alpha^{+}, \kappa, \vec{p}, \vec{D}\right] .
\end{array}\right.
$$

Since

$$
f_{\tau}\left(\alpha^{+}\right)=\alpha^{+} \cap \text { Skolem Hull }_{L^{++}}\left(\alpha \cup\left\{\alpha^{+}, \kappa, \bar{p}, \vec{D}\right\} \cup\left\{X_{i}: i<\tau\right\}\right),
$$

we have $\operatorname{dom}\left(g^{\alpha^{+}}\right)=f_{\tau}\left(\alpha^{+}\right)$. Set $g_{i}^{\alpha^{+}}=g^{\alpha^{+}}\left\lceil f_{i}\left(\alpha^{+}\right)\right.$. Then properties (1) and (2) above are clear. For (3), note that $\left\langle g_{j}^{\alpha^{+}}: \alpha^{+} \in \mathrm{CARD} \cap\left[\tau^{+}, \kappa\right)\right\rangle$ is (uniformly) definable in $X_{\tau^{+}(i+1)}$ from $X_{j}$. This uses the fact that for $\delta<f_{j}\left(\alpha^{+}\right)$, the $\triangleleft^{\alpha}$-least triple in (5.7) lies in $X_{j}$ on account of (5.6).

Claim. There exists a $p^{\prime} \leq \bar{p} \mid \tau^{+}$in $\mathbb{P}^{\tau^{+}}$such that if $p^{\prime} \in G$ and $G$ is $\mathbb{P}^{\tau^{+}}$generic over $L$, then

$$
X_{\alpha i}[G] \prec X_{\alpha j}[G] \prec L_{\kappa^{++}}[G]
$$

for all regular $\alpha \in \mathrm{CARD} \cap\left[\tau^{+}, \kappa\right)$ and all $i<j<\tau$.

Proof. If $\alpha>\omega_{1}$, then $\mathbb{P}^{\tau^{+}} \cup\left\{\mathbb{P}^{\tau^{+}}\right\} \subseteq X_{\alpha 0}$, using line (5.2) if $\tau>\omega$, and line (5.4) and $\omega_{1} \subseteq X_{\alpha 0}$ if $\tau=\omega$. The claim is immediate in this case.

On the other hand, if $\alpha=\omega_{1}$ and $\tau=\omega$, then by Lemma 2.3, there exists a $T^{\prime} \leq \bar{p}(\omega)$ in $\mathbb{S}$ such that

$$
T^{\prime} \Vdash X_{\omega_{1} i}[G] \prec X_{\omega_{1 j}}[G] \prec L_{\kappa^{++}}[G]
$$

for $i<j<\omega$. Let $p^{\prime} \in \mathbb{P}^{\omega_{1}}$ be such that $p^{\prime}(\omega)=T^{\prime}$. The claim is proved.

Without loss of generality, assume that $\bar{p} \mid \tau^{+}$itself is such a master condition.

Let $\stackrel{Q}{\tau}_{\tau}$ be a $\mathbb{P}^{\tau^{+}}$-term for a sparse subset of $\tau$ such that (for later convenience) $\bar{p} \mid \tau^{+} \Vdash 0 \in \stackrel{\circ}{\tau}_{\tau}$. We may assume that $\stackrel{\circ}{\tau}_{\tau}$ lies in $X_{\tau^{+} 0}$.

For $\alpha \in \mathrm{CARD} \cap[\tau, \kappa)$, let $\stackrel{\circ}{R}_{\alpha}$ be a (canonically chosen) $\mathbb{P}^{\alpha^{+}}$-term for an immune $\omega$-partition of $\alpha$. The point of making these choices canonically is insuring that $\left\langle\stackrel{\circ}{R}_{\alpha}: \alpha \in \mathrm{CARD} \cap[\tau, \kappa)\right\rangle \in X_{\tau^{+} 0}$.

We shall define $\left\langle p_{i}: i \leq \tau\right\rangle$, a sequence of conditions in $\mathbb{P}_{\tau^{+}}^{\kappa}$, such that

(1) $p_{0}=\bar{p} \uparrow\left[\tau^{+}, \kappa\right)$ and $\mathbb{P}^{\tau^{+}} \Vdash p_{i} \geq p_{j}$ if $i<j \leq \tau$;

(2) $p_{i}\left\lceil\alpha \Vdash \operatorname{ht}\left(p_{i}(\alpha)\right)=f_{i}(\alpha)\right.$ if $i>0$ and $\alpha \in \operatorname{sp}\left(p_{i}\right)$ is regular and uncountable; and 
(3) $\left\langle p_{j}: j \leq i\right\rangle \in X_{\tau^{+}(i+1)}$.

Begin by setting $p_{0}=\bar{p} \uparrow\left[\tau^{+}, \kappa\right)$.

Now suppose that $p_{i}$ has been defined. In defining $p_{i+1}$ there are two cases to consider. For simplicity, let us describe these cases as though we were working in a $\mathbb{P}^{\tau^{+}}$generic extension, keeping in mind that in fact we are describing elaborate $\mathbb{P}^{\tau^{+}}$-terms in $L$.

First of all, if $i \in Q_{\tau}$, choose $p_{i+1} \leq p_{i}$ to be $L$-least such that $p_{i+1}$ meets $D_{\mathrm{ot}\left(Q_{\tau} \cap i\right)}$ and $p_{i+1}\left\lceil\alpha \Vdash \operatorname{ht}\left(p_{i+1}(\alpha)\right)=f_{i+1}(\alpha)\right.$ for all uncountable regular $\alpha \in \operatorname{sp}\left(p_{i+1}\right)$. (Here ot $\left(Q_{\tau} \cap i\right)$ is the order-type of the set of ordinals $Q_{\tau} \cap i$.)

Note that $p_{i+1} \in X_{\tau^{+}(i+2)}$, since $p_{i} \in X_{\tau^{+}(i+1)}$ and $X_{\tau^{+}(i+2)}[G] \prec$ $L_{\kappa^{++}}[G]$ when $G$ is $\mathbb{P}^{\tau^{+}}$generic with $\bar{p} \mid \tau^{+} \in G$. This insures that $p_{i}^{L[G]}$ has an extension that lies in $X_{\tau^{+}(i+2)}[G]$ meeting $D_{\mathrm{ot}\left(Q_{\tau} \cap i\right)}^{L[G]}$. In fact, we have $\left\langle p_{j}: j \leq i+1\right\rangle \in X_{\tau^{+}(i+2)}$.

The other case to consider is that of $i \notin Q_{\tau}$. In this case, choose $p_{i+1} \leq p_{i}$ to be $L$-least such that $\operatorname{sp}\left(p_{i+1}\right)=\operatorname{sp}\left(p_{i}\right)$ and, for each uncountable regular $\alpha \in \operatorname{sp}\left(p_{i}\right)$, the condition $p_{i+1}\lceil\alpha$ forces

$$
\operatorname{ht}\left(p_{i+1}(\alpha)\right)=f_{i+1}(\alpha) \text { and } \quad p_{i+1}(\alpha)=(q, r),
$$

where

$$
\begin{aligned}
& q(\delta)=0 \text { for all } \delta \in\left[f_{i}(\alpha), f_{i+1}(\alpha)\right), \\
& r(\delta)=\left\langle\left\langle R_{f_{i+1}(\alpha)}(\delta), R_{\tau}(i)\right\rangle\right\rangle \text { if } \alpha \text { is inaccessible and } \delta \in\left[f_{i}(\alpha), f_{i+1}(\alpha)\right), \\
& r(\delta)=R_{\beta}\left(g_{i+1}^{\alpha}(\delta)\right) \text { if } \alpha=\beta^{+} \text {and } \delta \in\left[f_{i}(\alpha), f_{i+1}(\alpha)\right) .
\end{aligned}
$$

(Note that $p_{i}\left\lceil\alpha \Vdash \operatorname{ht}\left(p_{i}(\alpha)\right)=f_{i}(\alpha)\right.$ for uncountable regular $\alpha \in \operatorname{sp}\left(p_{i}\right)$, since $i \neq 0$ on account of our insistence that $0 \in Q_{\tau}$.)

Note also that $p_{i+1} \in X_{\tau^{+}(i+2)}$ and that, in fact, $\left\langle p_{j}: j \leq i+1\right\rangle \in$ $X_{\tau^{+}(i+2)}$. This uses that $\stackrel{\circ}{\tau}_{\tau},\left\langle\stackrel{\circ}{R}_{\alpha}: \alpha \in \operatorname{CARD} \cap[\tau, \kappa)\right\rangle \in X_{\tau^{+} 0}$ and that $\left\langle g_{j}^{\beta^{+}}: \beta \in \mathrm{CARD} \cap[\tau, \kappa)\right.$ and $\left.j \leq i+1\right\rangle \in X_{\tau^{+}(i+2)}$, as well as induction.

If $i$ is a limit ordinal, choose $p_{i}$ to be $L$-least such that

$$
p_{i}\left\lceil\alpha \Vdash p_{i}(\alpha)=\bigwedge_{j<i} p_{j}(\alpha),\right.
$$

where $\bigwedge_{j<i}\left(q_{j}, r_{j}\right)=\left(\bigcup_{j<i} q_{j}, \bigcup_{j<i} r_{j}\right)$. Then $p_{i}\left\lceil\alpha \Vdash \operatorname{ht}\left(p_{i}(\alpha)\right)=f_{i}(\alpha)\right.$ for all uncountable regular $\alpha \in \operatorname{sp}\left(p_{i}\right)$. The construction of $\left\langle p_{j}: j \leq i\right\rangle$ can be carried out inside $X_{\tau^{+}(i+1)}$. Hence $\left\langle p_{j}: j \leq i\right\rangle \in X_{\tau^{+}(i+1)}$. The only clause in the definition of $\mathbb{P}_{\tau^{+}}^{\kappa}$ which is not evident for $p_{i}$ is that $\mathbb{P}^{\alpha} \Vdash p_{i}(\alpha) \in \mathbb{Q}_{\alpha} \times \mathbb{R}_{\alpha}$ for $\alpha \in \operatorname{sp}\left(p_{i}\right)$. It suffices to see that $p_{i}\left\lceil\alpha \Vdash p_{i}(\alpha) \in \mathbb{Q}_{\alpha} \times \mathbb{R}_{\alpha}\right.$.

Let $H$ be $\mathbb{P}^{\alpha}$ generic over $L$ with $p_{i}\lceil\alpha \in H$. (So, formally, we are proceeding by induction on $\alpha$.) Work in $L[H]$. Say $p_{j}(\alpha)=\left(q_{j}, r_{j}\right)$ for $j<i$. 
We must argue that $q_{i}=\bigcup_{j<i} q_{j} \in \mathbb{Q}_{\alpha}$ and that $r_{i}=\bigcup_{j<i} r_{j} \in \mathbb{R}_{\alpha}$. Let $i_{0}<i$ be the least $j$ such that $\alpha \in \operatorname{sp}\left(p_{j}\right)$.

First we argue that $q_{i} \in \mathbb{Q}_{\alpha}$. Suppose that $y \subseteq f_{i}(\alpha)$ is infinite and constructible. We may assume that $y \cap f_{j}(\alpha)$ is finite for each $j<i$. Using the fact that $Q_{\tau} \subseteq \tau$ is sparse and that $\left\langle f_{i}(\alpha): i \leq \tau\right\rangle \in L$, we can find an infinite constructible $z \subseteq\left(y \backslash f_{i_{0}}(\alpha)\right)$ such that

$$
z \cap \bigcup_{j \in Q_{\tau}}\left[f_{j}(\alpha), f_{j+1}(\alpha)\right)=\emptyset
$$

But by construction,

$$
q_{i}^{-1}(1) \backslash f_{i_{0}}(\alpha) \subseteq \bigcup_{j \in Q_{\tau}}\left[f_{j}(\alpha), f_{j+1}(\alpha)\right) .
$$

Thus $z \cap q_{i}^{-1}(1)=\emptyset$, as required to verify that $q_{i} \in \mathbb{Q}_{\alpha}$.

Next we argue that $r_{i} \in \mathbb{R}_{\alpha}$. Suppose that $y \subseteq f_{i}(\alpha)$ is infinite and constructible. We must see that $r_{i}$ " $y$ is infinite. Again we may assume that $y \cap f_{j}(\alpha)$ is finite for each $j<i$. As before, first choose $z \subseteq\left(y \backslash f_{i_{0}}(\alpha)\right)$ to be infinite and constructible and as in (5.8). Then $z$ is an infinite constructible subset of $\bigcup_{j \notin Q_{\tau} \cup i_{0}}\left[f_{j}(\alpha), f_{j+1}(\alpha)\right)$. It suffices to see that $r_{i} " z$ is infinite. There are two cases to consider.

The first of these cases is that of inaccessible cardinals $\alpha$. By construction,

$$
r_{i} \uparrow \bigcup_{j \notin Q_{\tau} \cup i_{0}}\left[f_{j}(\alpha), f_{j+1}(\alpha)\right)=R \uparrow\left(f_{i}(\alpha) \cap \bigcup_{j \notin Q_{\tau} \cup i_{0}}\left[f_{j}(\alpha), f_{j+1}(\alpha)\right)\right),
$$

where $R(\delta)=\left\langle\left\langle R_{f_{j+1}(\alpha)}(\delta), R_{\tau}(j)\right\rangle\right\rangle$, for $\delta \in\left[f_{j}(\alpha), f_{j+1}(\alpha)\right)$. But $R_{f_{j+1}(\alpha)}$ is an immune $\omega$-partition of $f_{j+1}(\alpha)$ and $R_{\tau}$ is an immune $\omega$-partition of $\tau$. As we saw in the proof of Lemma 1.2, the function $R$ is an immune $\omega$-partition of $f_{\tau}(\alpha)$. It follows that $r_{i} " z$ is infinite.

The other case to consider is that of successor cardinals $\alpha$. Say $\alpha=\beta^{+}$. Then by construction,

$$
r_{i} \uparrow \bigcup_{j \notin Q_{\tau} \cup i_{0}}\left[f_{j}(\alpha), f_{j+1}(\alpha)\right)=\left(R_{\beta} \circ g^{\alpha}\right) \uparrow \bigcup_{j \notin Q_{\tau} \cup i_{0}}\left[f_{j}(\alpha), f_{j+1}(\alpha)\right) .
$$

Since $g^{\alpha}$ is one-to-one and constructible, and since $R_{\beta}$ is an immune $\omega$ partition, it follows that $r_{i} " z$ is infinite.

This completes the construction of $\left\langle p_{i}: i \leq \tau\right\rangle$.

If $G$ is $\mathbb{P}^{\tau^{+}}$generic with $\bar{p} \mid \tau^{+} \in G$, then $\stackrel{\circ}{Q}_{\tau}^{L[G]}$ is unbounded in the regular cardinal $\tau$. By construction, then, $p_{\tau}$ meets $\stackrel{\circ}{D}_{i}^{L[G]}$ for each $i<\tau$. The proof of the Distributivity Lemma is complete. 
Proof of the Density Lemma. We shall argue for (b). Then for (a) it suffices to see that $\mathbb{P}^{\omega_{2}}$ is dense in $\widehat{\mathbb{P}}^{\omega_{2}}$. This can be seen as below in the case $\kappa=\mu^{+}$, where $\mu>\tau^{+}$is regular.

If $\kappa$ is inaccessible, or if $\kappa=\mu^{+}$, where $\mu$ is singular, then our claim is immediate by induction.

Suppose now that $\kappa=\mu^{+}$, where $\mu>\tau^{+}$is regular. (The non-trivial case is $\mu=\lambda^{+}$, where $\lambda$ is singular.) Let $\stackrel{\circ}{q}$ and $\stackrel{\circ}{r}$ be $\widehat{\mathbb{P}}^{\mu}$-terms such that $\widehat{\mathbb{P}}^{\mu} \Vdash \bar{p}(\mu)=(\stackrel{\circ}{q}, \stackrel{\circ}{r})$. By induction, it suffices to find $\mathbb{P}^{\mu}$-terms $\stackrel{\circ}{q}^{\prime}$ and $\stackrel{\circ}{r}^{\prime}$, and a condition $p$ extending $\bar{p}\left\lceil\mu\right.$ in $\widehat{\mathbb{P}}_{\tau^{+}}^{\mu}$ such that

- $\stackrel{\circ}{q}^{\prime} \in L_{\mu}$ and $p \Vdash \stackrel{\circ}{q}=\stackrel{\circ}{q}$; and

- $\stackrel{\circ}{r}^{\prime} \in L_{\mu}$ and $p \Vdash \stackrel{\circ}{r}^{\prime}=\stackrel{\circ}{\text {. }}$.

First, choose $p^{\prime} \leq \bar{p}\left\lceil\mu\right.$ in $\widehat{\mathbb{P}}_{\tau^{+}}^{\mu}$ (that is, $\widehat{\mathbb{P}}^{\tau^{+}} \Vdash p^{\prime} \leq \bar{p}\left\lceil\mu\right.$ ) such that $p^{\prime} \Vdash$ $\operatorname{ht}(\bar{p}(\mu)) \leq \check{\beta}$ for some $\beta<\mu$. Then, for each $\gamma<\beta$, let $A_{\gamma} \subseteq \widehat{\mathbb{P}}^{\mu}$ be an antichain maximal among the set of conditions $p^{\prime \prime}$ such that if $p^{\prime \prime}$ is compatible with $p^{\prime}$, then $p^{\prime \prime} \Vdash \stackrel{q}{q}(\check{\gamma})=\check{\imath}$ for $i=0$ or $i=1$, and $p^{\prime \prime} \Vdash \stackrel{\circ}{r}(\check{\gamma})=\check{n}$ for some $n \in \omega$. By induction we may assume that in fact $A_{\gamma} \subseteq \mathbb{P}^{\mu}$. Now choose $p \leq p^{\prime}$ in $\widehat{\mathbb{P}}_{\tau^{+}}^{\mu}$ such that $\left|A_{\gamma}\right| p|\leq| \beta \mid$ for all $\gamma<\beta$. Then set

$$
\stackrel{\circ}{q}^{\prime}=\left\{\left((\gamma, i)^{\vee}, p^{\prime \prime}\right): p^{\prime \prime} \in A_{\gamma}\left\lceil p \text { and } p^{\prime \prime} \Vdash \stackrel{\circ}{q}(\check{\gamma})=\check{\imath}\right\}\right.
$$

and define $\dot{r}^{\prime}$ analogously. Note that since $A_{\gamma} \subseteq \mathbb{P}^{\mu} \subseteq L_{\mu}$ and $\left|A_{\gamma}\right| p|\leq| \beta \mid<$ $\mu$ and $\mu$ is regular, the terms $\stackrel{q}{q}^{\prime}$ and $\stackrel{\circ}{r}^{\prime}$ lie in $L_{\mu}$. By construction, $p \Vdash \stackrel{q}{q}^{\prime}=\stackrel{\circ}{q}$ and $p \Vdash \stackrel{\circ}{r}^{\prime}=\stackrel{\circ}{r}$.

Note next that if $\kappa=\mu^{+}$and $\mu=\tau^{+}$, then in the above construction, it is unnecessary to extend $\bar{p}\left\lceil\mu\right.$ (now in $\widehat{\mathbb{P}}_{\tau^{+}}^{\tau^{+}}=\{\emptyset\} !$ ) to force $\operatorname{ht}(\bar{p}(\mu)) \leq \beta$ for some $\beta<\mu$, or to reduce the antichains $A_{\gamma}$, because $\mathbb{P}^{\mu}=\mathbb{P}^{\tau^{+}} \subseteq L_{\tau}$.

Finally, suppose that $\kappa$ is singular. The construction in this case is a diagonal version of the proof of the Distributivity Lemma. Note that it suffices to handle the case $\tau \geq \operatorname{cf}(\kappa)$; the case $\tau<\operatorname{cf}(\kappa)$ then follows by induction.

Fix a condition $\bar{p}$ in $\widehat{\mathbb{P}}_{\tau^{+}}^{\kappa}$ and let $\lambda>\kappa^{+}$be a cardinal sufficiently large that $\bar{p} \in L_{\lambda}$ and that $\widehat{\mathbb{P}}^{\kappa} \cap L_{\lambda}$ is equivalent to $\widehat{\mathbb{P}}^{\kappa}$.

Let $\vec{\kappa}=\left\langle\kappa_{i}: i<\operatorname{cf}(\kappa)\right\rangle$ be a continuous, increasing sequence of cardinals that is cofinal in $\kappa$ with $\kappa_{0}=\tau^{+}$.

For $i<\operatorname{cf}(\kappa)$, set

$$
D_{i}=\left\{p \in \mathbb{P}_{\tau^{+}}^{\kappa_{i+1}}: \widehat{\mathbb{P}}^{\tau^{+}} \Vdash p \leq \bar{p} \uparrow\left[\tau^{+}, \kappa_{i+1}\right)\right\} .
$$

Of course, $D_{i}$ is not predense in general. However, if $p \in \mathbb{P}_{\tau^{+}}^{\kappa_{i}}$ and $\widehat{\mathbb{P}}^{\tau^{+}} \Vdash$ $p \leq \bar{p}\left\lceil\kappa_{i}\right.$, then, by induction, $p$ has an extension that lies in $D_{i}$. 
Define the Skolem hulls $X_{\alpha i}$ as in the proof of the Distributivity Lemma, only taking hulls inside $L_{\lambda^{+}}$and including the parameters $\vec{\kappa}$ and $\widehat{\mathbb{P}}^{\kappa} \cap L_{\lambda}$. Then define $f_{i}$ and the $g_{i}^{\alpha^{+}}$as in the proof of the Distributivity Lemma.

Finally, define $\left\langle p_{i}: i \leq \operatorname{cf}(\kappa)\right\rangle$ as in the proof of the Distributivity Lemma, except setting $p_{0}=\emptyset$. (So at stages $i \in Q_{\tau}$, the condition $p_{i+1}$ is chosen to extend both $p_{i}$ and $\bar{p} \mid \kappa_{i+1}$, and at stages $i \notin Q_{\tau}$, we have $\operatorname{sp}\left(p_{i+1}\right)=\operatorname{sp}\left(p_{i}\right)$.) Then $p=\bigwedge_{i<\operatorname{cf}(\kappa)} p_{i}$ is a condition in $\mathbb{P}_{\tau^{+}}^{\kappa}$ such that $\widehat{\mathbb{P}} \tau^{+} \Vdash p \leq \bar{p}$.

6. One more time. Let us now turn to the class version $\mathbb{R}_{\infty}$ of $\mathbb{R}_{\kappa}$, defined in a $\widehat{\mathbb{P}}$ generic extension of $L$. The purpose of $\mathbb{R}_{\infty}$ is finally to add an immune $\omega$-partition of the ordinals.

Working in a $\widehat{\mathbb{P}}$ generic extension of $L$, let $\mathbb{R}_{\infty}$ consist of all functions $r: \alpha \rightarrow \omega$, for some ordinal $\alpha$, such that if $x \subseteq \alpha$ is infinite and constructible, then $r " x$ is infinite. Let $\mathbb{R}_{\infty}$ be ordered by reverse set inclusion, that is, by reverse functional extension. Set $\widehat{\mathbb{P}}^{+}=\widehat{\mathbb{P}} * \mathbb{R}_{\infty}$.

Using the fact that $\widehat{\mathbb{P}}$ adds an immune $\omega$-partition to each uncountable cardinal $\alpha$, the following extension lemma is clear.

Lemma 6.1. Assume that $(\bar{p}, \bar{r}) \in \widehat{\mathbb{P}}^{+}$and that $\bar{p} \Vdash \operatorname{dom}(\bar{r})=\check{\alpha}$. Suppose $\delta>\alpha$. Then there exists a term $r \in \mathbb{R}_{\infty}$ such that $(\bar{p}, \bar{r}) \geq(\bar{p}, r)$ and $\bar{p} \Vdash \operatorname{dom}(r)=\check{\delta}$.

It is evident, then, that if $G * H$ is $\widehat{\mathbb{P}}^{+}$generic over $L$, then

$$
R_{\infty}=\left\{r^{L[G]}:(p, r) \in G * H \text { for some } p\right\}
$$

is an immune $\omega$-partition of the ordinals.

Since $\mathbb{R}_{\infty}$ adds an amenable class to a $\widehat{\mathbb{P}}$ generic extension, it is immediate that $\widehat{\mathbb{P}}^{+}$is cardinal and GCH preserving. It remains to check that $\widehat{\mathbb{P}}^{+}$ preserves ZFC.

Working in $L$, define the sub-ordering $\mathbb{P}^{+}$of $\widehat{\mathbb{P}}^{+}$to consist of all pairs $(p, r)$ such that

(1) $p \in \mathbb{P}=\bigcup_{\kappa \in \mathrm{CARD}} \mathbb{P}^{\kappa}$, and

(2) $r$ is a $\mathbb{P}$-term and $\mathbb{P} \Vdash r \in \mathbb{R}_{\infty}$.

Then $\mathbb{P}^{+}$is a dense subclass of $\widehat{\mathbb{P}}^{+}$. Let $\mathbb{P}_{\tau^{+}}^{+}$consist of those pairs $(p, r)$ in $\mathbb{P}^{+}$such that $\operatorname{sp}(p) \cap \tau^{+}=\emptyset$. By the Density Lemma in $\S 5, \mathbb{P}_{\tau^{+}}^{+}$is dense in $\widehat{\mathbb{P}}_{\tau^{+}}^{+}$, when $\tau$ is regular.

In order to verify that $\widehat{\mathbb{P}}^{+}$preserves ZFC, it suffices to establish this

Metalemma 6.2. Suppose $\tau$ is regular. Then

$$
\mathbb{P}^{\tau^{+}} \Vdash " \mathbb{P}_{\tau^{+}} * \mathbb{R}_{\infty} \text { is definably } \leq \tau \text {-distributive". }
$$


That is, if $\left\langle\stackrel{\circ}{D}_{i}: i\langle\tau\rangle\right.$ is a uniformly definable sequence of $\mathbb{P}^{\tau^{+}}$-terms for predense classes in $\mathbb{P}_{\tau^{+}} * \mathbb{R}_{\infty}$ and if $(\bar{p}, \bar{r}) \in \mathbb{P}^{+}$, then there exists a condition $(p, r) \leq(\bar{p}, \bar{r})$ such that

$$
p\left\lceil\tau^{+} \Vdash "\left(p \uparrow\left[\tau^{+}, \infty\right), r\right) \text { meets } \stackrel{\circ}{D}_{i} "\right.
$$

for all $i<\tau$.

Proof sketch. The proof of this lemma is similar to that of the Distributivity Lemma in $\S 5$. Let us outline the necessary modifications of that proof.

Suppose $\left\langle\stackrel{\circ}{D}_{i}: i<\tau\right\rangle$ is uniformly definable over $L$ from the parameter $a$. Begin by defining

$\alpha_{i}=$ the least $\alpha$ such that $\tau \cup\{\tau, \bar{p}, a\} \cup\left\{\alpha_{j}: j<i\right\} \subseteq L_{\alpha}$ and $L_{\alpha} \prec_{\Sigma_{n}} L$, where $n \geq 2$ is sufficiently large. Then, in the notation of the Distributivity Lemma, set $X_{i}=L_{\alpha_{i}}$. Set

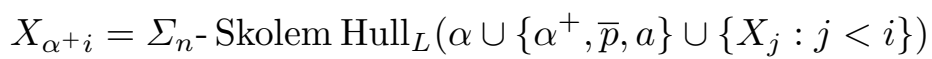

and, if $\alpha$ is inaccessible, set

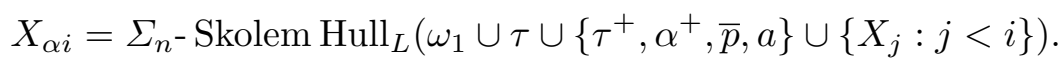

The proof then proceeds as does that of the Distributivity Lemma, mutatis mutandis. (For example, instances of $\prec$ are replaced with $\prec_{\Sigma_{n}}$ and, in the definition of $g^{\alpha^{+}}$, the formulas $\varphi$ are restricted to being $\Sigma_{n}$ formulas.)

This completes the proof of the first sentence of Theorem 3. Adding a Cohen real over a $\widehat{\mathbb{P}}^{+}$generic extension suffices for the first sentence of Theorem 2. To establish the second parts of these theorems what remains is constructing $\mathbb{P}$ generic classes in $L\left[0^{\#}\right]$.

7. The construction from $0^{\#}$. Working in $L\left[0^{\#}\right]$, our goal is to define a class $G$ that is $\mathbb{P}$ generic over $L$. If $\alpha$ is $L$-regular, let $R_{\alpha}$ denote the (canonical) immune $\omega$-partition of $\alpha$ added by this generic class. Then $G$ will have the following coherence property:

$$
R_{\bar{\iota}}=R_{\iota} \uparrow \bar{\iota} \quad \text { whenever } \bar{\iota}<\iota \text { are Silver indiscernibles. }
$$

Let $\mathcal{I}$ denote the class of Silver indiscernibles. Then $R_{\infty}=\bigcup_{\iota \in \mathcal{I}} R_{\iota}$ will be an $L\left[0^{\#}\right]$-definable $\omega$-partition of the ordinals that is amenable to an $L$-cardinal preserving inner model. It follows that $R_{\infty}$ is as required for the second part of Theorem 3 . Since $\omega_{1}^{L\left[R_{\infty}\right]}=\omega_{1}^{L}<\omega_{1}^{L\left[0^{\#}\right]}$, in $L\left[0^{\#}\right]$ there exist reals that are Cohen generic over $L\left[R_{\infty}\right]$. The second part of Theorem 2 follows.

It is not difficult to see that $G *\left\{R_{\infty}\lceil\alpha: \alpha \in \mathrm{OR}\}\right.$ is $\mathbb{P}^{+}$generic over $L$. However, we shall not need this fact. 
Some ad hoc conventions will be useful in this section. We shall use $\vec{\alpha}, \vec{\beta}$, and so forth, to denote increasing finite sequences of ordinals $\left\langle\alpha_{0}, \ldots, \alpha_{k}\right\rangle$ where each $\alpha_{i}$ is either $\omega_{1}$ or is an $L$-inaccessible cardinal. We shall use $\alpha^{+}$ to denote the least $L$-cardinal greater than $\alpha$, and $\alpha^{*}$ to denote the least indiscernible greater than $\alpha$.

By recursion on $|\vec{\alpha}| \geq 2$ we shall define conditions $p^{\vec{\alpha}}$ in such a way that

(1) $p^{\vec{\alpha}} \in \mathbb{P}_{\alpha_{0}^{+}}^{\alpha_{1}^{+}}$;

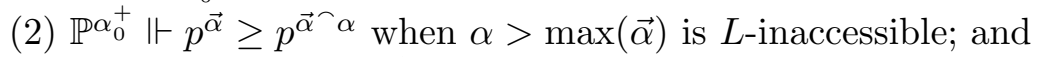

(3) the function $\vec{\alpha} \mapsto p^{\vec{\alpha}}$ is definable without parameters over $L$.

Then a $\mathbb{P}_{\omega_{2}}$ generic class will be generated by the conditions $p^{\vec{\alpha}}$ for $\vec{\alpha} \in$ $\left(\left\{\omega_{1}\right\} \cup \mathcal{I}\right)^{<\omega}$. The definition of the $p^{\vec{\alpha}}$ 's, and hence of this generic class, is quite easy. The work to be done in this section is in verifying that this class is indeed $\mathbb{P}_{\omega_{2}}$ generic.

If $|\vec{\alpha}|=2$, then choose $p^{\vec{\alpha}}$ to be $L$-least such that

(a) $\operatorname{sp}\left(p^{\vec{\alpha}}\right)=\left\{\alpha_{1}\right\}$, and

(b) $\mathbb{P}_{0}^{\alpha_{0}^{+}} \Vdash p^{\vec{\alpha}}\left(\alpha_{1}\right)=\left(\bigcup \stackrel{\circ}{Q}_{\alpha_{0}}, \bigcup \stackrel{\circ}{R}_{\alpha_{0}}\right)$,

where $\stackrel{\circ}{Q}_{\alpha_{0}}$ and $\stackrel{\circ}{R}_{\alpha_{0}}$ are terms for $\mathbb{Q}_{\alpha_{0}}$ and $\mathbb{R}_{\alpha_{0}}$ generic objects, respectively.

If $\alpha>\max (\vec{\alpha})$ is $L$-inaccessible, choose $p^{\vec{\alpha}^{-} \alpha}$ to be $L$-least such that

(a) $\mathbb{P}_{0}^{\alpha_{0}^{+}} \Vdash p^{\vec{\alpha}} \geq p^{\vec{\alpha}^{-} \alpha}$, and

(b) $\mathbb{P}^{\alpha_{0}^{+}} \Vdash p^{\vec{\alpha}^{\wedge} \alpha} \in \stackrel{\circ}{D}$,

where $\stackrel{D}{D}$ is a term such that if $H$ is $\mathbb{P}_{0}^{+}$generic over $L$, then

$$
\begin{aligned}
& \stackrel{\circ}{D}^{L[H]}=\bigcap\left\{\stackrel{\circ}{X}^{L[H]}: \stackrel{\circ}{X} \in \text { Skolem Hull }_{L_{\alpha}}\left(\alpha_{0} \cup\{\vec{\alpha}\}\right)\right. \text { and } \\
& \dot{\circ}^{L[H]} \text { is a dense open subset of } \mathbb{P}_{\alpha_{0}^{+}}^{\alpha_{1}^{+}} \text {. }
\end{aligned}
$$

Since $\mathbb{P}^{\alpha_{0}^{+}} \Vdash{ }^{\alpha_{1}^{+}}{ }_{\alpha_{0}^{+}}^{\alpha^{+}}$is $\leq \alpha_{0}$-distributive", such a condition $p^{\vec{\alpha}^{\wedge} \alpha}$ exists. This completes the definition of $p^{\vec{\alpha}}$.

Suppose that $\alpha<\beta$ and that $D \subseteq \mathbb{P}^{\beta}$ is constructible and predense. Then a condition $p \in \mathbb{P}_{\alpha}^{\beta}$ reduces $D$ below $\alpha$ iff there exists a (constructible) predense $D^{\prime} \subseteq \mathbb{P}^{\alpha}$ such that $p^{\prime} \cup p$ meets $D$ whenever $p^{\prime} \in D^{\prime}$.

Claim 1. Suppose $\iota \in \mathcal{I}$ and that $D \subseteq \mathbb{P}^{\iota^{+}}$is a constructible predense set. Then there exists an $\vec{\alpha} \in\left(\left\{\omega_{1}\right\} \cup \mathcal{I}\right)^{<\omega}$ with $\alpha_{1}=\iota$ such that $p^{\vec{\alpha}}$ reduces $D$ below $\alpha_{0}^{+}$.

P r o o f. Working in $L$, let us begin by making a general observation about predensity reduction in $\mathbb{P}$. Assume that $\tau$ is an uncountable regular cardinal and that $\kappa>\tau$ is a cardinal. Suppose that $D \subseteq \mathbb{P}^{\kappa}$ is predense and that $\bar{p} \in \mathbb{P}_{\tau^{+}}^{\kappa}$. Then there exists a condition $p \in \mathbb{P}_{\tau^{+}}^{\kappa}$ such that $\mathbb{P}^{\tau^{+}} \Vdash p \leq \bar{p}$ and 
$p$ reduces $D$ below $\tau^{+}$. Indeed, it is a general fact about full iterations that there exists $p^{\prime} \in \widehat{\mathbb{P}}_{\tau^{+}}^{\kappa}$ such that $p^{\prime}$ reduces $D$ below $\tau^{+}$and $\widehat{\mathbb{P}}_{\tau^{+}}^{\kappa} \Vdash p^{\prime} \leq \bar{p}$. (Simply construct a $\widehat{\mathbb{P}}^{\tau^{+}}$-term with this property.) But then by the Density Lemma, there exists a condition $p \in \mathbb{P}_{\tau^{+}}^{\kappa}$ as required extending $p^{\prime}$.

Now suppose that $D \subseteq \mathbb{P}^{\iota^{+}}$is a constructible predense set. Set $\alpha_{1}=\iota$ and choose $\alpha_{0}$ and $\alpha_{2}, \ldots, \alpha_{k+1}$ from $\left\{\omega_{1}\right\} \cup \mathcal{I}$ such that $\alpha_{0}<\alpha_{1}<\alpha_{2}<$ $\cdots<\alpha_{k+1}$ and $D$ lies in the Skolem hull in $L_{\alpha_{k+1}}$ of $\alpha_{0} \cup\left\{\alpha_{0}, \ldots, \alpha_{k}\right\}$. Let $\stackrel{\circ}{X}$ be a $\mathbb{P}_{0}^{+}$-term in this Skolem hull for

$$
\left\{p \in \mathbb{P}_{\alpha_{0}^{+}}^{\alpha_{1}^{+}}: p \text { reduces } D \text { below } \alpha_{0}^{+}\right\} .
$$

Then $\mathbb{P}_{0}^{\alpha_{0}^{+}} \Vdash$ " $X \stackrel{\circ}{ }$ is dense and open". Setting $\vec{\alpha}=\left\langle\alpha_{0}, \ldots, \alpha_{k}\right\rangle$, we have $\mathbb{P}^{\alpha_{0}^{+}} \Vdash p^{\vec{\alpha}^{-} \alpha_{k+1}} \in \stackrel{\circ}{X}$.

Claim 2. Suppose that $\vec{\alpha}^{0}, \ldots, \vec{\alpha}^{n} \in\left(\left\{\omega_{1}\right\} \cup \mathcal{I}\right)^{<\omega}$. Then the conditions $p^{\vec{\alpha}^{0}}, \ldots, p^{\vec{\alpha}^{n}}$ have a common extension in $\mathbb{P}$.

Proof. The proof is really just an exercise in indiscernibility using two features of the current setting, namely, the coherence enforced by our definition of $p^{\vec{\alpha}}$ when $|\vec{\alpha}|=2$, and the fact that $p(\alpha) \in L_{\alpha}$ when $\alpha>\omega$.

We may assume that $\left|\vec{\alpha}^{i}\right|=\left|\vec{\alpha}^{j}\right|$ for all $i, j \leq n$. Note first that $\operatorname{sp}\left(p^{\vec{\alpha}^{i}}\right) \subseteq$ $\left(\alpha_{0}^{i}, \alpha_{0}^{i *}\right) \cup\left\{\alpha_{1}^{i}\right\}$ by indiscernibility. Also by indiscernibility we may assume that there exists a fixed sequence $\vec{\iota} \in \mathcal{I}^{<\omega}$ such that $\vec{\alpha}^{i}=\left\langle\alpha_{0}^{i}, \alpha_{1}^{i}, \vec{\iota}\right\rangle$ for all $i \leq n$.

Choose a finite set $B \subseteq\left\{\omega_{1}\right\} \cup \mathcal{I}$ such that each of $\vec{\alpha}^{0}, \ldots, \vec{\alpha}^{n}$ lies in

$$
\left\{\left\langle\beta_{0}, \beta_{1}, \vec{\iota}\right\rangle: \beta_{0}<\beta_{1} \text { lie in } B\right\} \text {. }
$$

And let $\left\langle\beta_{0}, \ldots, \beta_{k}\right\rangle$ enumerate $B$ in increasing order.

We maintain that the condition $p$ defined by

$$
p=\bigcup_{i<k} p^{\left\langle\beta_{i}, \beta_{i+1}, \vec{l}\right\rangle}
$$

is a common extension of all of $p^{\vec{\alpha}^{0}}, \ldots, p^{\vec{\alpha}^{n}}$. In fact, fixing $i \leq k$, let us argue that

$$
p \leq p^{\left\langle\beta_{j}, \beta_{i}, \vec{\imath}\right\rangle} \quad \text { for all } j<i .
$$

This is trivial if $i=0$, so suppose that $i>0$. If $j<i$, then

$$
\operatorname{sp}\left(p^{\left\langle\beta_{j}, \beta_{i}, \vec{\iota}\right\rangle}\right) \subseteq\left(\beta_{j}, \beta_{j+1}\right) \cup\left\{\beta_{i}\right\},
$$

and by indiscernibility

$$
p^{\left\langle\beta_{j}, \beta_{i}, \vec{L}\right\rangle} \uparrow\left(\beta_{j}, \beta_{j+1}\right)=p^{\left\langle\beta_{j}, \beta_{j+1}, \vec{l}\right\rangle} \uparrow\left(\beta_{j}, \beta_{j+1}\right)=p \uparrow\left(\beta_{j}, \beta_{j+1}\right) .
$$

So $p\left\lceil\beta_{i} \leq p^{\left\langle\beta_{j}, \beta_{i}, \vec{\iota}\right\rangle} \uparrow \beta_{i}\right.$ for all $j<i$. 
Now proceed by induction on $i-j$, for $i>j \geq 0$, to see that

$$
p\left\lceil\beta_{i} \Vdash p^{\left\langle\beta_{j}, \beta_{i}, \vec{c}\right\rangle}\left(\beta_{i}\right) \geq p^{\left\langle\beta_{i-1}, \beta_{i}, \vec{\imath}\right\rangle}\left(\beta_{i}\right) .\right.
$$

Because $p\left(\beta_{i}\right)=p^{\left\langle\beta_{i-1}, \beta_{i}, \vec{c}\right\rangle}\left(\beta_{i}\right)$, this suffices to finish the proof of (7.2).

If $j=i-1$, then this claim is trivial. Suppose that $0 \leq j<i-1$. Note first that by indiscernibility,

$$
p^{\left\langle\beta_{j}, \beta_{i}, \vec{\iota}\right\rangle}\left(\beta_{i}\right)=p^{\left\langle\beta_{j}, \beta_{j+1}, \vec{\iota}\right\rangle}\left(\beta_{j+1}\right) .
$$

So

$$
p^{\left\langle\beta_{j}, \beta_{j+1}, \vec{\iota}\right\rangle} \uparrow \beta_{j+1} \Vdash p^{\left\langle\beta_{j}, \beta_{i}, \vec{\imath}\right\rangle}\left(\beta_{i}\right) \in \stackrel{\circ}{Q}_{\beta_{j+1}} \times \stackrel{\circ}{R}_{\beta_{j+1}} .
$$

Also,

$$
p^{\left\langle\beta_{j+1}, \beta_{i}, \vec{l}\right\rangle} \uparrow \beta_{i} \Vdash \forall(q, r) \in \stackrel{\circ}{Q}_{\beta_{j+1}} \times \stackrel{\circ}{R}_{\beta_{j+1}}(q, r) \geq p^{\left\langle\beta_{j+1}, \beta_{i}, \vec{c}\right\rangle}\left(\beta_{i}\right),
$$

by the definition of $p^{\left\langle\beta_{j+1}, \beta_{i}\right\rangle}$.

Now

and

$$
p\left\lceil\beta_{i} \leq p\left\lceil\beta_{j+1} \leq p^{\left\langle\beta_{j}, \beta_{j+1}, \vec{l}\right\rangle} \uparrow \beta_{j+1}\right.\right.
$$

$$
p\left\lceil\beta_{i} \leq p \uparrow\left(\beta_{j+1}, \beta_{j+2}\right)=p^{\left\langle\beta_{j+1}, \beta_{j+2}, \vec{l}\right\rangle}\left\lceil\beta_{j+2}=p^{\left\langle\beta_{j+1}, \beta_{i}, \vec{l}\right\rangle} \uparrow \beta_{i} .\right.\right.
$$

Hence from (7.3) and (7.4) we obtain

$$
p\left\lceil\beta_{i} \Vdash p^{\left\langle\beta_{j}, \beta_{i}, \vec{\iota}\right\rangle}\left(\beta_{i}\right) \geq p^{\left\langle\beta_{j+1}, \beta_{i}, \vec{\iota}\right\rangle}\left(\beta_{i}\right)\right.
$$

and so by induction

$$
p\left\lceil\beta_{i} \Vdash p^{\left\langle\beta_{j}, \beta_{i}, \vec{\iota}\right\rangle}\left(\beta_{i}\right) \geq p^{\left\langle\beta_{i-1}, \beta_{i}, \vec{\imath}\right\rangle}\left(\beta_{i}\right) .\right.
$$

If $X \subseteq \mathbb{P}$, define $\operatorname{Gen}(X)$ by

$$
\begin{aligned}
p \in \operatorname{Gen}(X) \text { iff } & \text { there exist conditions } p_{1}, \ldots, p_{n} \in X \text { such that } \\
& \text { if } p^{\prime} \leq p_{1}, \ldots, p_{n}, \text { then } p^{\prime} \leq p .
\end{aligned}
$$

Choose $H \subseteq \mathbb{P}_{2}^{\omega_{2}^{L}}$ to be $\mathbb{P}_{2}^{\omega_{2}^{L}}$ generic over $L$. Then set

$$
G=\operatorname{Gen}\left(H \cup\left\{p^{\vec{\alpha}}: \vec{\alpha} \in\left(\left\{\omega_{1}\right\} \cup \mathcal{I}\right)^{<\omega}\right\}\right) .
$$

We maintain that $G$ is $\mathbb{P}$ generic over $L$. It is evident from the definition of $p^{\vec{\alpha}}$ when $|\vec{\alpha}|=2$ that $G$ has the coherence property (7.1). Thus showing $G$ to be $\mathbb{P}$ generic over $L$ will complete the proofs of Theorems 2 and 3 .

Certainly, if $p \in G$ and $\bar{p} \geq p$, then $\bar{p} \in G$. It follows from Claim 2 (and the fact that $\omega, \omega_{1} \notin \operatorname{sp}\left(p^{\vec{\alpha}}\right)$ for $\vec{\alpha} \in\left(\left\{\omega_{1}\right\} \cup \mathcal{I}\right)^{<\omega}$ ) that $G$ consists of pairwise compatible conditions. Thus it suffices to see that $G \cap A \neq \emptyset$ whenever $A \subseteq \mathbb{P}$ is an $L$-definable maximal antichain.

First note that if $A \subseteq \mathbb{P}$ is an $L$-definable antichain, then $A \in L$. This observation uses the facts that conditions in $\mathbb{P}$ have Easton support and that in the presence of $0^{\#}$ there exist unboundedly many $L$-Mahlo cardinals $\alpha$ such that $L_{\alpha} \prec L$. 
So suppose $A \in L$ is a maximal antichain in $\mathbb{P}$. Then $A$ is a maximal antichain in $\mathbb{P}^{\iota}$ for some $\iota \in \mathcal{I}$, hence a maximal antichain in $\mathbb{P}^{\iota^{+}}$. It follows from Claim 1 and the well-foundedness of the ordinals that there exist finitely many $\vec{\alpha}^{1}, \ldots, \vec{\alpha}^{n} \in\left(\left\{\omega_{1}\right\} \cup \mathcal{I}\right)^{<\omega}$ such that if $p^{\vec{\alpha}^{1}}, \ldots, p^{\vec{\alpha}^{n}} \geq p$, then $p$ reduces $A$ below $\omega_{2}^{L}$. Since $H$ is $\mathbb{P}^{\omega_{2}^{L}}$ generic over $L$, we conclude that $G \cap A \neq \emptyset$.

Remark. It is evident from the construction of $G$ that if $j: L \rightarrow L$ is an elementary endomorphism, then $p \in G$ iff $j(p) \in G$. It follows that the Silver indiscernibles remain indiscernible in $L[G]$ (and $L\left[R_{\infty}\right]$, for that matter).

8. Questions. It is possible to explore in at least two directions, namely, (1) supplying appropriate definitions and considering immune $\kappa$-partitions for $\kappa>\omega$, and (2) considering immune partitions of unordered $n$-tuples of ordinals for $1<n<\omega$. Some questions can be settled by adapting the proof of Theorems 2 and 3.

For example, suppose $1<\kappa \leq \omega$ and $1 \leq n<\omega$ and define an $n$ immune $\kappa$-partition of a set (class) $X$ to be a function $F:[X]^{n} \rightarrow \kappa$ such that if $x \subseteq X$ is infinite and constructible, then $\left|F^{\prime \prime}[x]^{n}\right|=\kappa$. The immune partitions discussed heretofore are 1-immune partitions in this terminology. Let us briefly consider 2-immune partitions.

It is not difficult to see that 2-immune partitions can be added generically by essentially the same method as 1-immune partitions. For example, the following forcing operations add a 2-immune 2-partition of the ordinals:

(a) Add sparse subsets to $\omega$ and $\omega_{1}$, say using Sacks forcing and $\mathbb{Q}_{\omega_{1}}$.

(b) Add a sparse subset to each regular $\alpha>\omega_{1}$ and add a 2-immune $\omega$-partition of the ordinals, using an adaptation of the backwards Easton support iteration $\widehat{\mathbb{P}}^{+}$. At regular cardinals $\alpha>\omega_{1}$, add a 2 -immune $\omega$-partition of $\alpha$ with conditions that are 2-immune $\omega$ partitions of ordinals $\delta<\alpha$. Notice that there is no need to add any new subsets of $\omega_{1}$ on account of $F:\left[\omega_{1}\right]^{2} \rightarrow \omega$, where $F(\{\alpha<\beta\})=$ $f_{\beta}(\alpha)$ and $f_{\beta}$ projects $\beta$ into $\omega$.

(c) Add a Cohen real to obtain a 2-immune 2-partition of the ordinals.

In fact, adding a 1-immune 2-partition of the ordinals adds a 2-immune 2-partition of the ordinals: If $F: \mu \rightarrow \kappa$ is a 1-immune $\kappa$-partition, then $G:[\mu]^{2} \rightarrow \kappa$ defined by $G(\{\alpha<\beta\})=F(\beta)$ is a 2 -immune $\kappa$-partition. The converse is open.

Matters to explore include:

(1) Does the existence of an $(n+2)$-immune $\kappa$-partition ever imply the existence of an $(n+1)$-immune $\kappa$-partition? 
(2) Is there anything interesting to say about immune $\kappa$-partitions for $\kappa>\omega ?$

(3) Is there anything interesting to say about immunity relativized to inner models other than $L$ ?

(4) (Sy Friedman in $[\mathrm{F}]$ ) Suppose that $1<\kappa \leq \omega$. We say $F: X \rightarrow \kappa$ is weakly immune iff $|F " x|>1$ whenever $x \subseteq X$ is infinite. Does the existence of a weakly immune $\kappa$-partition, where $2<\kappa<\omega$, imply the existence of an immune 2-partition?

We say that an immune $\kappa$-partition $F$ : OR $\rightarrow \kappa$ is minimal iff $F$ is definable over $L[x]$ whenever $x$ is an infinite set of ordinals with the property that $|F " x|<\kappa$.

The $\omega$-partition of Theorem 3 satisfies a weak version of this property. Suppose that $1<\kappa \leq \omega$ and $F:$ OR $\rightarrow \kappa$ is an immune $\kappa$-partition of the ordinals. We say that $F$ is weakly minimal when there exists a fixed non-constructible real $r$ such that $r \in L[x]$ whenever $x \subseteq$ OR is infinite and $\left|F^{\prime \prime} x\right|<\kappa$.

THEOREM 4. There exists a weakly minimal immune $\omega$-partition of the ordinals in a cardinal and GCH preserving generic extension of L. If $0^{\#}$ exists, then such a generic extension of $L$ is definable over $L\left[0^{\#}\right]$.

Proof. The forcing property $\widehat{\mathbb{P}}^{+}$is equivalent to $\mathbb{S} * \widehat{\mathbb{P}}_{\omega_{1}}^{+}$, where $\mathbb{S}$ is Sacks forcing below the condition $T_{*}$. Work in a $\widehat{\mathbb{P}}^{+}$generic extension. Fix a Sacks real $r$. Suppose $x$ is an infinite set of ordinals, but that $R_{\infty}$ " $x$ is finite. We may assume that $x$ is countable. Since $\mathbb{S} \Vdash ~ “ \widehat{\mathbb{P}}_{\omega_{1}}^{+}$is $\leq \omega$-distributive", we deduce that $x \in L[r]$ and that $x$ is non-constructible. Thus $r \in L[x]$.

It remains open whether it is consistent that there exists a minimal immune partition of the ordinals.

\section{References}

[F] S. Friedman, An immune partition of the ordinals, in: Recursion Theory Week, Lecture Notes in Math. 1144, Springer, New York, 1986, 141-147.

DEPARTMENT OF MATHEMATICS AND COMPUTER SCIENCE

SAN JOSE STATE UNIVERSITY

SAN JOSE, CALIFORNIA 95192-0103

U.S.A.

E-mail: STANLEY@MATH.SJSU.EDU 